\title{
Usefulness of a Transconjunctival Approach in the Reconstruction of the Medial Blow-Out Wall Fracture
}

Chi An Lee,

Hook Sun, Ji Young Yun

Department of Plastic and Reconstructive Surgery, Inje University Busan Paik Hospital, Inje University School of Medicine, Busan, Korea

No potential conflict of interest relevant to this article was reported.

\begin{abstract}
Background: A transcaruncular approach is typically used for reconstructions of medial wall fractures. However, others reported that a transconjunctival approach was conducive for securing an adequate surgical field of view. In this study, we aimed to examine the extent of repair of medial wall fracture via a transconjunctival approach. Methods: We retrospectively reviewed the medical records of 50 patients diagnosed as having medial wall fracture via preoperative computed tomography and who underwent surgery between March 2011 and February 2014. The fracture location was defined by dividing each of the anterior-posterior and superior-inferior distances into three compartments.

Results: A transcaruncular approach was used in 7 patients, while the transconjunctival approach was performed in the remaining 43 patients. The transconjunctival approach enabled a relatively broad range of repair that partially included the front and back of the medial wall, and was successful in $86 \%$ of the entire study population.

Conclusion: It is known that more than $50 \%$ of total cases of the medial wall fracture occur mainly in the middle-middle portion, a majority of which can be reconstructed via a transconjunctival approach. We used a transconjunctival approach in identifying the location of the fracture on image scans except for cases including the fracture of the superior portion in patients with medial wall fracture. If it is possible to identify the location of the fracture, a transconjunctival approach would be an useful method for the reconstruction in that it causes no damages to the lacrimal system and is useful in confirming the overall status of the floor.
\end{abstract}

Keywords: Orbital fracture / Reconstructive surgical procedure / Ophthalmologic surgical procedure / Orbital implants

\section{INTRODUCTION}

The medial wall forms an almost rectangular shape. It extends from the anterior lacrimal crest to the orbital apex. Moreover, it articulates with the roof at the fronto-ethmoid suture and the floor at the maxillo-ethmoidl suture [1]. It has the thickest portion in the most posterior part that has the connection to the optic canal. In the adjacent areas to lamina papyracea located central to it, there are bony structures that are as thin as a paper. This makes it

\section{Correspondence: Hook Sun}

Department of Plastic and Reconstructive Surgery, Inje University Busan Paik Hospital, Inje University School of Medicine, 75 Bokji-ro, Busanjin-gu, Busan 47392, Korea E-mail: sun443@hanmail.net

Received March 28, 2017 / Revised May 25, 2017 / Accepted May 25, 2017 vulnerable to even a blunt trauma and infections.

Such methods as a transcaruncular approach and Lynch incision are commonly used for the surgical repair of the medial wall fracture. Moreover, both a subcilliary incision and a transconjunctival approach are commonly used for the repair of floor fracture (Fig. 1). Kim et al. [2] reported that Lynch incision would not be appropriate, although it has been conventionally used, in the surgical repair of the medial wall fracture because it provides a small amount of visual field and causes noticeable scars. Thus, they proposed a transcaruncular approach be used as an alternative method. According to Ridgway et al. [3], a transconjunctival approach is useful in securing wider surgical fields and causes no scars. These authors also noted that it causes almost no eyelid re- 


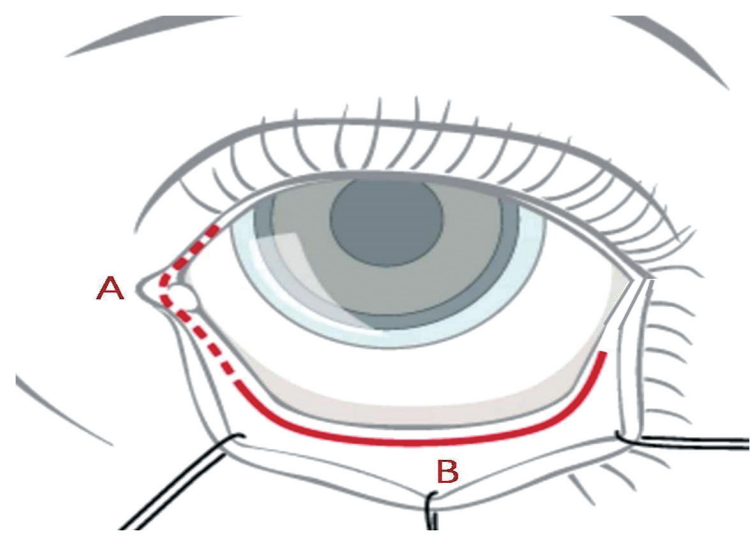

Fig. 1. Surgical approach for medial orbital fracture. A, transcruncular approach (vertical incision through lateral one third of the caruncle, about $1 \mathrm{~cm}$ ); $\mathrm{B}$, transconjunctival approach (horizontal incision at 3-4 mm inferior to lower eyelid margin, about $2.5 \mathrm{~cm}$ ).

traction that may occur during a subcilliary approach. According to Nowinski et al. [4], the incidence of ectropion and lower lid retraction is relatively lower in cases of the floor fracture. Thus, these authors advocated the use of a transconjunctival approach as a method of choice.

To date, a transcaruncular approach has been conventionally used for the surgical repair of the medial wall fracture. By contrast, Novelli et al. [5] reported that a transconjunctival approach was useful in sufficiently securing operative fields without causing damages to the lacrimal system and medial canthus in patients with medial wall fracture. Moreover, orbital fractures commonly occur as a result of blunt trauma to the facial region. Furthermore, floor fractures are concurrently present in 7\%-53\% of total cases of the medial wall fracture [6].

We divided the medial wall into specific compartments in cases of medial wall fracture and thereby examined the incidence of fractures depending on the location. In addition, we performed a surgical repair of the medial wall fracture and examined the extent of the surgical repair via a transconjunctival approach.

\section{METHODS}

We retrospectively reviewed the medical records of 50 patients who preoperatively had a medial wall fracture on computed tomography $(\mathrm{CT})$ and underwent surgery for the fracture between
March 2011 and February 2014. This study was reviewed and approved by the Ethics Review Board of the InJe University Health Center (IRB no. 17-0020). Our clinical series of patients consist of 38 men and 12 women, whose mean age was 37.2 years old (range, 11-70 years old). The patients with concurrently fractures of other facial structures, such as nasal bone, maxilla, zygomatic bone, mandible and frontal sinus, were excluded. Moreover, the patients who concurrently had orbital floor fractures on CT scans after sustaining an injury were also excluded.

A preoperative diagnosis was made on CT scans, by which the location of the medial wall fracture was measured. On axial views of CT scans, the depth of the fracture was measured. On coronal views of CT scans, the height of the fracture was measured. Lacrimal crest was located on the anterior side. The opening of optic canal was located on the posterior side. The areas where a slope was formed between the roof and floor of the medial wall were served as landmarks of the superior and inferior portions. Distances extending from the anterior to posterior directions and those extending from the superior to inferior directions were divided into three parts. Thus, a total of nine compartments were served. Based on these compartments, the location of the fracture was determined (Fig. 2).

All the patients were surgically treated under general anesthesia. Following the insertion of a corneal protector, epinephrine was added to a mixture of $0.9 \%$ normal saline and $2 \%$ lidocaine at a ratio of 1:200,000 for the purposes of promoting the hemostasis. This was followed by the infiltration. To rule out the tarsal plane, a double-hooked retractor was placed to the medial side of the margin of the lower eyelid and the eversion was done accordingly. This was followed by an incision made at 3-4 $\mathrm{mm}$ inferior lower eyelid margin, using a needle-tipped electrocautery. A conjunctival incision was made involving the inferior lacrimal pumtum to medial directions. These maneuvers were performed using the retroseptal technique. Using a metzenbaum, the dissection was done towards the inferior orbital rim.

Using an eletrocautery, the periosteum was dissected. Thus, the inferior orbital rim and orbital floor were exposed. Moreover, the medial wall was also carefully exposed. Using a malleable retractor, the herniation of the periorbital fat was prevented. 
Once the fracture was identified, the reduction was done as maximally as possible. Using porous polyethylene incorporating titanium or other materials, the reconstruction was performed. However In the patients whose medial wall fracture could not be identified, a transcaruncular incision was additionally made for the surgical reconstruction. Following the reconstruction, the periosteum was sutured using the 6-0 Vicryl. Moreover, the conjunctiva was sutured using the 8-0 Vicryl. At the time, the buried suture was performed to minimize the foreign body sensations.

Postoperatively, the antibiotic and steroid eye drops were applied to the conjunctiva for more than 5 days.

\section{RESULTS}

Of all the patients, $52 \%$ concurrently had fractures of middlemiddle portion. In $26 \%$ of total patients, there were fractures in wide areas involving more than two compartments. Based on both a superior and inferior classification and an anterior and posterior one, fractures of the middle portion were predominantly prevalent. Fractures were more prevalent by five times in the anterior portion as compared with the posterior one. Moreover, fractures were more prevalent by nearly three times in the inferior portion as compared with the superior one (Fig. 3).

\section{Superior}

\begin{tabular}{|c|c|c|c|}
\hline \multirow{3}{*}{ Anterior } & $\begin{array}{c}2 \\
(4)\end{array}$ & $\begin{array}{c}2 \\
(4)\end{array}$ & $\begin{array}{c}1 \\
\text { (2) }\end{array}$ \\
\hline & $\begin{array}{c}14 \\
(28)\end{array}$ & $\begin{array}{c}26 \\
(52)\end{array}$ & $\begin{array}{c}2 \\
\text { (4) }\end{array}$ \\
\hline & $\begin{array}{l}4 \\
(8)\end{array}$ & $\begin{array}{c}9 \\
(18)\end{array}$ & $\begin{array}{l}1 \\
\text { (2) }\end{array}$ \\
\hline
\end{tabular}

Posterior

\section{Inferior}

Fig. 2. A schematic illustration of the compartmentation of the medial wall. Distances extending from the anterior to posterior directions and form the superior to inferior ones were divided into three parts.

\begin{tabular}{l|l|l|l|}
\multicolumn{4}{|c|}{ Superior } \\
\cline { 2 - 4 } & Anterosuperior & Middle-superior & Posterosuperior \\
\cline { 2 - 4 } & Middle-anterior & Middle-middle & Middle-posteior \\
\cline { 2 - 4 } & Anteroinferior & Middle-inferior & Posteroinferior \\
\hline \multicolumn{3}{|c|}{ Inferior }
\end{tabular}

Fig. 3. The number and proportion of the patients with fractures depending on the compartment. Above is the munber of patients with proportion in brackets below. Middle-middle portion, anterior-middle portion and middle-inferior portionshowed predominant prevalence. 
In our series, a transtcaruncular approach was used for the surgical repair of seven cases. It was appropriate for the upper $1 / 3$ and middle-posterior part of the medial wall. On the other hand, a transconjunctival approach was used for the reconstruction in the remaining 43 cases (Figs. 4, 5). In addition, we also successfully performed the reconstruction in the patients with slight diffuse fracture lines that are superior or posterior to the middleanterior and middle-middle portion. This indicates that the extent of a transconjunctival approach was so great as to involve some parts of the superior and posterior side in the reconstruc-

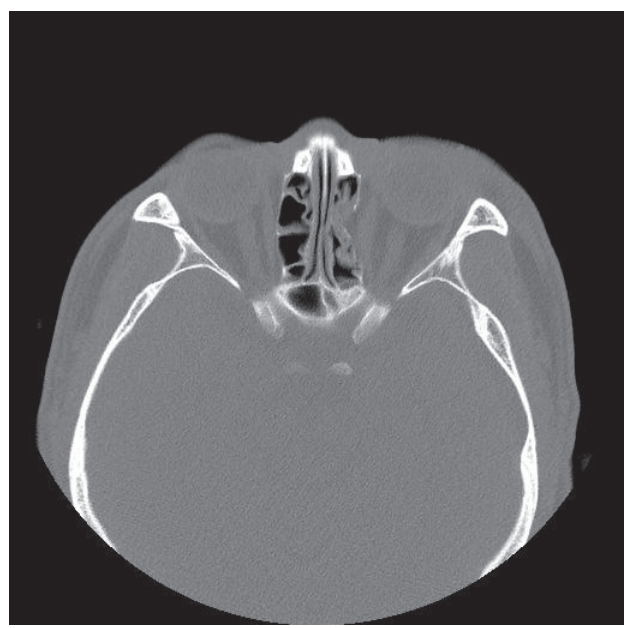

Fig. 4. Preoperative computed tomography scan. Axial views of preoperative computed tomography scans in the patients who were diagnosed with medial wall fracture. tion of the medial wall fracture; this was seen in $86 \%$ of total patients (Fig. 6).

Of the 50 patients, 5 were confirmed to have fractures of the floor, although they were diagnosed with only the medial wall fracture on CT scans. Four patients had linear fracture lines on orbital floor, but one patient was performed reconstruction operation with an orbital implant.

In our series, there were no long-term complications such as the exposure of the placed implant, infections at surgical sites or foreign body reactions. In two patients who postoperatively had

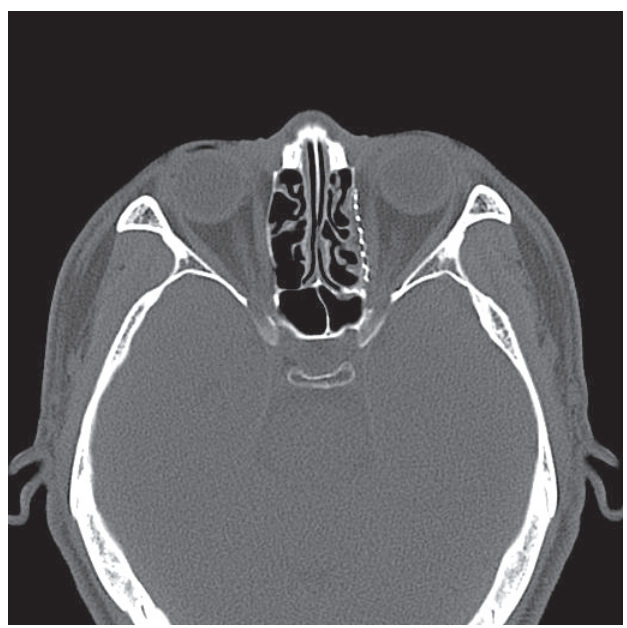

Fig. 5. Postoperative computed tomography scan. Axial views of postoperative computed tomography scans. Porous polyethylene incorporating titanium was used for the reconstruction of the medial wall fracture.

\section{Superior}

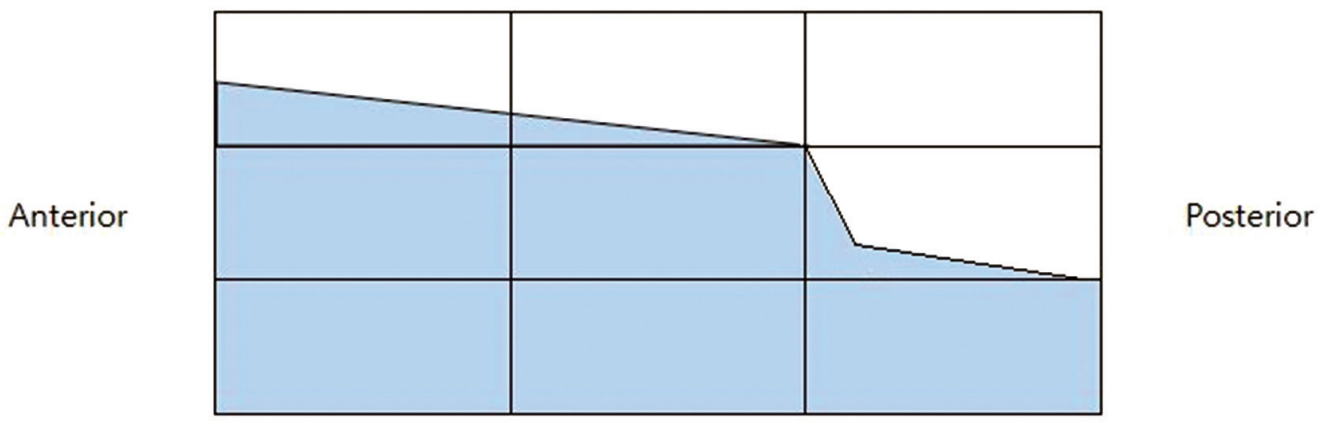

Inferior

Fig. 6. The extent of surgical approach via a transconjunctival approach. The extent of a transconjunctival approach was so great as to involve some parts of the superior and posterior side in the reconstruction of the medial wall fracture; this was seen in $86 \%$ of total patients. 
Table 1. The number of the patients who developed postoperative complications

\begin{tabular}{lc} 
Complication & No. \\
Early & \\
Ectropion & 2 \\
Foreign body sensation & 11 \\
Late & \\
Enophthalmos & 3 \\
\hline
\end{tabular}

enophthalmos, secondary operations were performed at 1 month after surgery. These patients also exhibited no further complications.

In our series, there were 13 cases of short-term complications, such as two cases of ectropion due to swelling, within 1-2 days postoperatively and 11 cases of foreign body sensations due to the suture of the conjunctiva. But this was recovered through conservative treatments. Therefore, such patients were not in need of further surgial procedures (Table 1).

\section{DISCUSSION}

Of the 50 patients, 26 had fractures of the central compartment of nine ones. This indicates that the orbital wall around the lamina papyracea is a fragile structure. In addition, fractures of the superior and posterior portion were notably less prevalent as compared with opposite cases. This suggests that the area connecting between the posterior part of the lacrimal crest and the orbital floor is a fragile one. This supports the fact that the floor of the medial wall is a thin structure in the inferior orbital fissure and it is vulnerable to the fracture due to a blow [1]

It has been reported that a transcaruncular approach is a more advantageous modality as compared with Lynch incision in patients with pure medial wall fracture [7-10]. Its advantages include a lack of postoperative scars, a lower possibility of damage to the lacrimal system and no notable difference in the operative field from the percutaneous method. Nevertheless, the possibility of damage to the lacrimal system (lacrimal sac injury, inferior canalicular obstruction with epiphora) cannot be completely ruled out. And transcaruncular approach can cause complications such as medial canthus detachment and inferior oblique dysfunction [5,11].
A transcaruncular approach is useful in securing a sufficient amount of operative field extending from the maxilloethmoidal strut to the frontoethmoidal suture in the surgical repair of the medial wall [7-9]. It is commonly encountered, however, patients with blunt orbital trauma concurrently have fractures of the medial wall and floor. But such patients are not indicated in the surgical repair via a transcaruncular approach, which is one of its disadvantages.

Unlike these features, a transconjunctival approach has the following three advantages: First, it provides adequate access for the reconstruction of the medial wall. Although it is difficult to approach the superior portion and middle-posterior portion, fracture frequency of these areas are rare. With the exception of fractures in such areas with low accessibility, almost $90 \%$ of all medial fractures can be reconstructed via a transconjunctival approach.

Second, it enables the surgeon to assess the floor status. Medial wall fractures are frequently accompanied by floor fractures, and floor fractures were intraoperatively discovered in about $10 \%$ of cases in which only a medial wall fracture was confirmed via preoperative CT. This indicates that a transconjunctival approach is a useful modality in directly confirming the status of the floor in the reconstruction of the medial wall fracture. It is also advantageous in confirming the status of sites where the medial wall fracture prevalently occurs in the reconstruction of the floor fracture.

Third, it poses no risk of damage to the lacrimal system. The lacrimal punctum or canaliculi are not damaged because the incision is only made up to the punctum. This ensures that the lacrimal punctum or canaliculi are left intact. Unlike a transcaruncular approach that may cause damages to such structures, a transconjunctival one is advantageous in maintaining a higher degree of their anatomical stability.

To summarize, we found that a transconjunctival approach was as useful as a transcaruncular approach for treatment of medial orbital fracture, except for cases including the fracture of the superior and middle-posterior portion. If it is possible to identify the location of the fracture, a transconjunctival approach would be an appropriate method for the reconstruction in that it causes no damages to the lacrimal system and is useful in confirming the overall status of the floor. 


\section{REFERENCES}

1. Rene C. Update on orbital anatomy. Eye (Lond) 2006;20:1119-29.

2. Kim S, Helen Lew M, Chung SH, Kook K, Juan Y, Lee S. Repair of medial orbital wall fracture: transcaruncular approach. Orbit 2005;24:1-9.

3. Ridgway EB, Chen C, Lee BT. Acquired entropion associated with the transconjunctival incision for facial fracture management. J Craniofac Surg 2009;20:1412-5.

4. Nowinski D, Messo E, Hedlund A. Treatment of orbital fractures: evaluation of surgical techniques and materials for reconstruction. J Craniofac Surg 2010;21:1033-7.

5. Novelli G, Ferrari L, Sozzi D, Mazzoleni F, Bozzetti A. Transconjunctival approach in orbital traumatology: a review of 56 cases. J Craniomaxillofac Surg 2011;39:266-70.
6. Thiagarajah C, Kersten RC. Medial wall fracture: an update. Craniomaxillofac Trauma Reconstr 2009;2:135-9.

7. You HJ, Kim DW, Dhong ES, Yoon ES. Precaruncular approach for the reconstruction of medial orbital wall fractures. Ann Plast Surg 2014;72:652-6.

8. Lee K, Snape L. Efficacy of Transcaruncular approach to reconstruct isolated medial orbital fracture. J Maxillofac Oral Surg 2010;9:142-5.

9. Shorr N, Baylis HI, Goldberg RA, Perry JD. Transcaruncular approach to the medial orbit and orbital apex. Ophthalmology 2000;107:1459-63.

10. Oh JY, Rah SH, Kim YH. Transcaruncular approach to blowout fractures of the medial orbital wall. Korean J Ophthalmol 2003;17:50-4.

11. Choi M, Flores RL. Medial orbital wall fractures and the transcaruncular approach. J Craniofac Surg 2012;23:696-701. 